\title{
Design of Information Interaction System Based on Android and JAVA EE architecture
}

\author{
Hongxia Liu \\ Department of Electronic Information Engineering, Handan Polytechnic College, Handan, 056001, \\ China \\ liuhongxia@163.com
} Keywords: Android system; JAVA EE architecture; Information interaction; Least squares;
Database

\begin{abstract}
Android system is currently the most frequently used smart mobile operating system, lightweight JAVA EE architecture has good stability, extensibility and compatibility, therefore it has been widely applied in the development and design of system. In this paper, information interaction platform based on the communicative community interaction has been designed, using Android intelligent mobile system and JAVA EE architecture, and optimization design was carried out on the information network system by using the least squares algorithm, generally mobile web page has been converted into mobile portable devices using JAVA programming technology. In the end, the development environment and the interface of the system for the information interaction platform has been set up. It is found that system platform can run smoothly through the mobile phone testing of Android4.0 above version, which provides a theoretical reference and technical support for the design of the interactive information database.
\end{abstract}

\section{Introduction}

The mobile Internet Based on the Android is is one of the most attractive prospects, the largest market potential and the fastest growing industry in recent years. As now the biggest sales of intelligent mobile operating system, Android is at the forefront of the wave of mobile internet now., but also in the forefront of the mobile Internet wave. People in communication community accept new things faster and faster in current society, and they is eager to demand more and more interaction information for others, so it has a broad prospect that provide portable interactive system based on Android for communication community.

The abbreviation of lightweight Java EE standard is SSH. Unlike traditional Java EE platform need the support of the EJB server, the application based on lightweight Java EE platform can run in ordinary Web server, and SSH has excellent stability and expansibility and maintainability. In this paper, the information interaction system based on the Android intelligent system and JAVA $\mathrm{EE}$ architecture has been designed using the SSH platform of, the main design process is shown in figure 1.

The general process of information interaction system has been shown in Figure 1. As shown in figure 1 in this paper, we use Android intelligent mobile system and use the Web server as compatible server., combined with the JAVA EE framework for system development. Using MyEclipse 9.0 application integration environment, the application system database is obtained by integration development at last, on the basis of the database we can complete the development of information interaction system. 


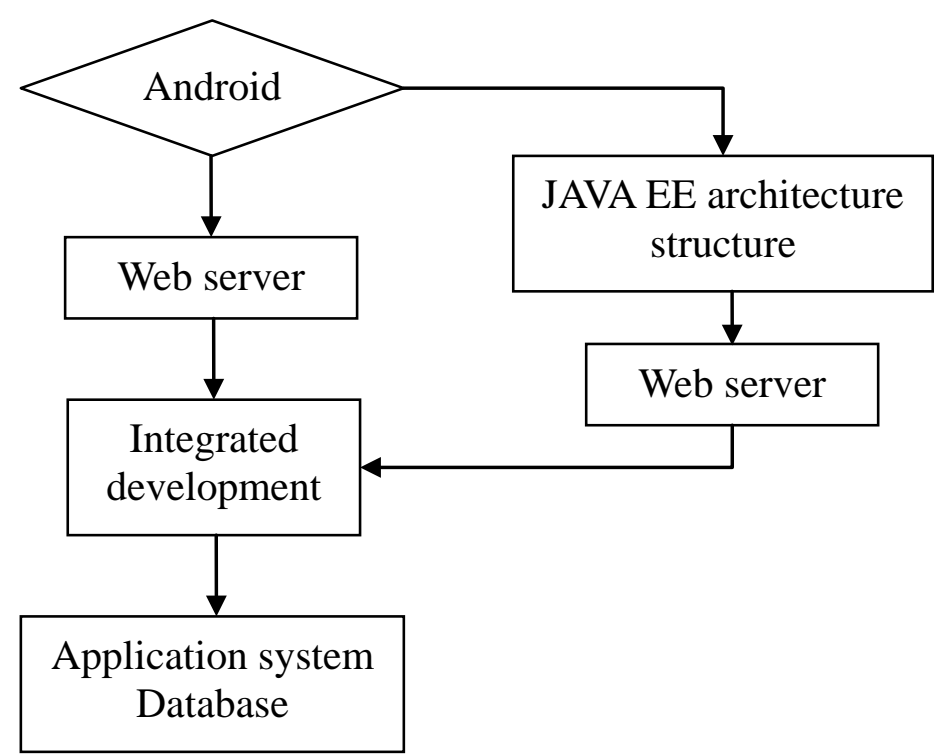

Fig.1 The main design process of information interaction system

\section{The information network optimization algorithm design based on the Android and JAVA EE architecture}

In development and design of android mobile information network system, the network node processing of JAVA EE architecture is one of the most key factors to improve network performance, we use the least squares algorithm to optimize design in the paper. First we assume that the node array of JAVA architecture is $\left(x_{1}, s_{1}\right),\left(x_{2}, s_{3}\right) \ldots \ldots .\left(x_{n}, s_{n}\right)$, then we can get the formula (1).

$s_{i}=\lambda+n x_{i}+X_{\sigma i}$

The node $\mathrm{n}$ value can be optimized by least square algorithm, the estimate value can be written as shown in formula (2).

$$
\widehat{n}=\frac{\sum_{i=1}^{m}\left(x_{i}-\bar{x}\right)\left(s_{i}-\bar{s}\right)}{\sum_{i=1}^{m}\left(x_{i}-\bar{x}\right)^{2}}
$$

In formula (2),

$\bar{X}=\frac{1}{m} \sum_{i=1}^{m} x_{i}^{\prime} \bar{s}=\frac{1}{m} \sum_{i=1}^{m} s_{i}$

$X_{\sigma}$ JAVA node maximum estimated likelihood value of $\sigma$ in can be shown in formula (4),

$\hat{\sigma}=\sqrt{\frac{1}{m} \sum_{i=1}^{m} x_{\sigma_{i}}{ }^{2}}$

After optimization of JAVA node $Y_{d}$, the node density can be got as shown in formula (5),

$$
f\left(Y_{d}\right)=\frac{1}{\hat{\sigma} \sqrt{2 \pi}} e^{\frac{-\left[Y_{d}-\left(P_{f}+Z_{f}+Z_{j}-Y_{l}-Y_{d}\right)\right]}{2 \sigma^{2}}}
$$

When $S_{d} \geq S d_{0}$, the network architecture can achieve optimal performance. Through the network optimization, and Android browsers have more perfect, we can make use of JAVA program the ordinary web content will be automatically converted to suitable for mobile phone screen display style, its main algorithm procedure is as follows,

Intent intent=new intent();

String data="http://bbs.jar.edu.cn/index2.html”

Uri uri=Uri.parse(data);

Intent setAction(intent.ACTION_VIEW); 
Intent setData(uri);

Start Activity(intent);

\section{Design of Information Interaction System Based on Android and JAVA EE architecture}

In order to verify the validity and reliability of the network optimization algorithm based the Android and JAVA EE architecture in the second section, in this section we uses the JAVA EE framework and Android system to built the information interaction platform, the system development environment of the main configuration is as follows.

Tab.1 System development environment table

\begin{tabular}{|c|c|}
\hline Client & Android 4.0 \\
\hline Server & $\begin{array}{c}\text { Servlet3.0+Hibernate3.0+Spring3.0.5 } \\
\text { Integration application environment MyEclipse 9.0 }\end{array}$ \\
\hline JDK & JDK1.6.3 \\
\hline Web server & apache tomcat 7.0.19 \\
\hline Application database & mysql 5.5.15 \\
\hline
\end{tabular}

The main development environment settings of interactive information system is shown in Table 1, campus information interaction system of Android + JAVA EE architecture is divided into client and server, in which the Android 4.0 copyright is mainly used in the client, and mysql5.5.15 database is mainly used in the server.

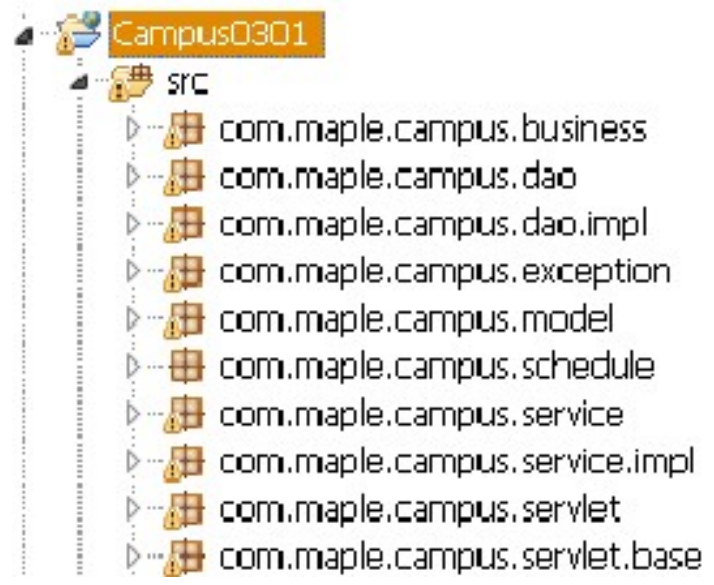

Fig.2 Schematic diagram of jars package intuitive way

The diagram of relevant Jar package used in the paper is shown in Figure 2, the intuitive way of Jar package and system files needed for system development can be more intuitive express in the figure, which include many structure, and the application of the Android system structure is shown in figure 3.

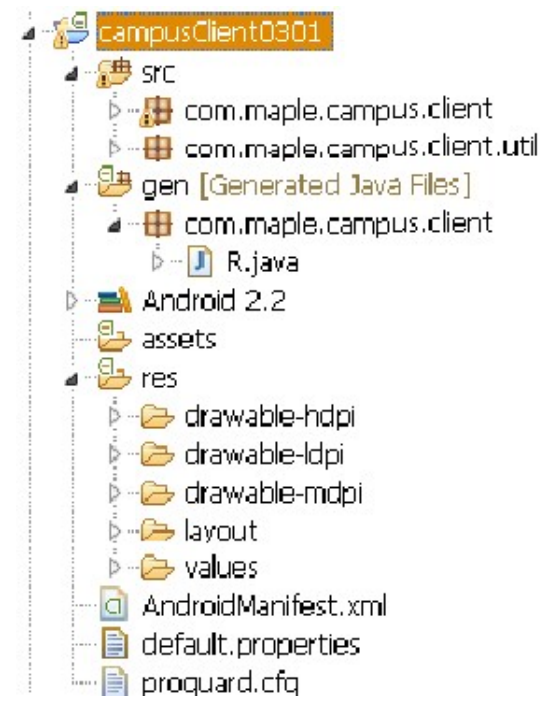

Fig.3 The application layer structure 
As shown in figure 3, Src directory is used to hold the Java source files in the above file structure, including Android component files Activity etc.. Res directory is used to hold various resource file of Android projects, the vast majority of custom resource files are XML format.

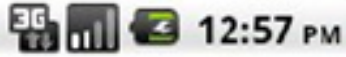

The login information interaction system

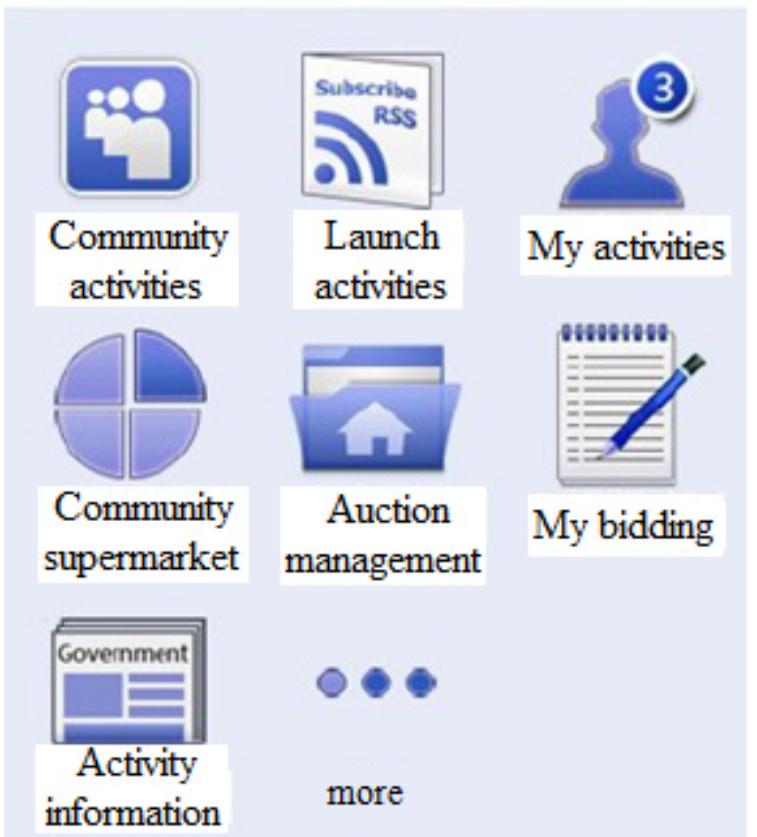

Fig.4 The community information interaction system interface

The community information system interface developed by JAVA EE framework and Android system is shown in figure 4 . Because the system is used as mobile portable devices, the interface pays more attention to the user experience than ordinary JAVA interface, the simple and beautiful of interface is one of the major consideration in interface development.

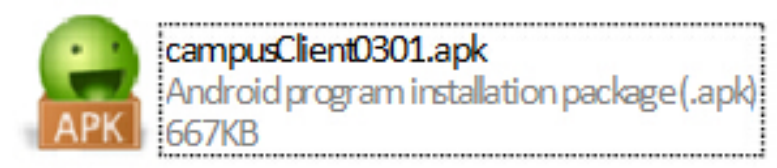

Fig. 5 The Android file installation package

As shown in figure 5, it will be generated Android installation file of .apk format under the bin folder of the project directory, after the completion of the client development and running through the Eclipse. It is found that the system can run smoothly through the Android4.0 above version of the mobile phone testing, which verifies the validity and reliability in the process of Android applications system development using the JAVA EE architecture.

\section{Conclusion}

The JAVA EE optimization algorithm of network architecture using the least squares method has been designed in this paper, and the compatibility of Web server and the Android intelligent mobile system has been enhanced by using the JAVA programming method, then information interaction system based on communication community has been built. The information interactive platform has been designed by using Android4.0 version and MySQL database, which adopts the JAVA EE architecture to make the system has better scalability and compatibility.

Through Android4.0 above version of the mobile phone testing, it found that the system platform to run smoothly, and the system interface is simple and beautiful, which can be accepted by the public and can be applied in the interactive system. 


\section{References}

[1] Li Qian, Ji Xiaoming, Wang Mingliang. Virtual human simulation method based on Java3D. Journal of Computer applications, Vol. 30 (11), (2010), p.3084-3086

[2]Kang Wenke, Zhang Dinaghua, Yu Suihuai. Color analyzer key methods of color conversion. Computer engineering and application, Vol. 46-48 (1), (2010), p.51-53

[3] Jiang Yu, Du Bin, Lu Jun etc. Algorithm of drawing isoline based on Delaunay triangulation net. Computer application research In, Vol. 27 (1), (2010), p.2010-103

[4] Yao Yuming, Liu Weiguo. Study of Android's Architecture and application development. Computer system application, Vol.11,(2009),p.37-39.

[5] Aida Niknejad. A Quality Evaluation of an Android Smartphone Application. Eastern Michigan University, (2011).

[6] Froehlich, Christopher. The Complete Idiot 's Guide to The Android App Development. ALPHA BOOKS, (2011).

[7] Liu Changping, Fan Mingyu, Wang Guangwei etc. Lightweight access control oriented toward Android. Application Research of Computers, (2011), Vol.27, p.25-29.

[8] Gong Lei. Zhou Cong. Development and research of mobile terminal application based on Android. Computer and Modernization, Vol. 7, (2008), p.85-89.

[10] Hoog, Andrew. Android Forensics: study, Analysis and Mobile Security for Google Android. ALPHA BOOKS, (2010).

[11] Li Xiaopeng. Electronic wallet system design and security research based on NFC technology. Beijing: Beijing university of posts and telecommunications, (2007)

[12] Xia Wendong, Lin Kai. 3G smart card system Fusion of NFC. Computer engineering,Vol.37, (2011), p.230-239 